\title{
Análisis de errores en el uso del artículo definido por sinohablantes
}

Estefany Isabel Macías Rioja*

Resumen: Esta ponencia tiene como fin presentar el trabajo de investigación para obtener el título de Licenciatura de la carrera de Lengua y Literaturas Hispánicas de la Facultad de Filosofía y Letras de la Universidad Nacional Autónoma de México (UNAM). En este trabajo se abordó el uso del artículo definido en español por hablantes cuya lengua materna es el chino. Con el fin de describir y analizar los usos del artículo definido por sinohablantes, se levantó un corpus conformado por redacciones y traducciones de oraciones de chino a español entre estudiantes del Centro de Enseñanza para Extranjeros (CEPE) de la UNAM e informantes que concluyeron o abandonaron los estudios del español y llevan varios años residiendo en México. Asimismo, presentamos el significado del artículo definido en español y los usos que fueron más representativos en nuestro corpus. Además, explicamos brevemente a qué nos referimos con el término chino, y abordamos algunas características de esta lengua para poder explicar cómo se expresa la definitud ya que esta lengua carece de artículos. Finalmente se presentan los hallazgos más relevantes de la investigación, tanto en los errores, como en los aciertos de nuestros informantes, y algunos aspectos que pueden estudiarse en el futuro.

Palabras clave: artículo definido, unicidad, chino, definitud en chino, sinohablantes, análisis de errores.

Summary: This paper aims to present the research work for the degree of Bachelor of major in Hispanic Language and Literature from Faculty of Arts of the National Autonomous University of Mexico (UNAM). Therefore, this paper addressed the use of the definite article in Spanish by speakers whose first language is Chinese. In order to describe and analyze the use of the definite article by Chinese speakers, we get a corpus that is composed of essays and translations from Chinese to Spanish sentences among students from Centro de Enseñanza para Extranjeros (CEPE) of UNAM and informants who completed or dropped out the their Spanish lessons and spent several years living in Mexico. We also present the meaning of the definite article in Spanish and its most representative uses in our corpus. Furthermore, we briefly explain what we mean by Chinese term and address some features of this language to explain how definiteness is, since this language

* Profesora de español del Centro de Enseñanza para Extranjeros 
lacks articles. Finally we present the most important findings of the investigation, both the errors, as in the successes of our informants, and certain aspects that can be studied in the future.

Keywords: definited article, uniqueness, Chinese, Chinese definiteness, Chinese speakers, error analysis.

Dentro del contraste entre el español y el chino mandarín, uno de los rasgos que se ha comentado y estudiado es la existencia del artículo definido en el primero y la ausencia de este determinante en el segundo (Lin: 2003). Por lo tanto, es interesante ver las repercusiones de este hecho en hablantes cuya lengua e el chino mandarín cuando están en el proceso del estudio de español, e incluso cuando ya concluyeron o abandonaron los estudios formales, es decir en una escuela, de esta lengua. Las preguntas que surgen a partir de este contraste son ¿cuáles son los errores que cometen?, ¿en qué contextos?, ¿se llega a dominar en niveles avanzados? y la pregunta que no se debe dejar de lado es ¿cuáles son los aciertos en el uso de este determinante?

Paracomenzar el estudio de este tema, hay que considerar no sólola morfología del artículo definido en español (el, la, los, las) y la relación de concordancia de género y número que mantiene con el sustantivo al que antecede, sino también los rasgos semántico o pragmático que aporta al sintagma nominal para poder dar una explicación acerca de su uso. En este sentido, los conceptos principales que caracterizan a este determinante son definitud y unicidad.

La definitud significa que el referente, es decir, el objeto real aludido por el sustantivo que conforma el sintagma nominal (SN), puede ser identificado unívocamente por el oyente (Leonetti 1999:794). En este sentido, se ha insistido que la presencia del artículo definido indica que el referente al que precede es conocido por el oyente (Alcina y Franch 1988:550), (Gili Gaya 1980:\$184:243). Sin embargo, la presencia del artículo definido también es necesaria cuando se introduce información nueva para el oyente cuya identificación en el discurso o contexto comunicativo es accesible, es decir, es fácil de identificar (Leonetti 1999a:\$12.1.1.3.).

En cuanto a la unicidad, este concepto implica que el uso del artículo definido es necesario debido a que el referente puede ser identificado de manera unívoca, es decir, que corresponde a un referente único. Por ejemplo, los sintagmas nominales (SSNN) definidos como el Sol, la Luna y la Tierra, tienen referentes unívocos porque designan a seres únicos; no hay otros candidatos que cumplan con sus características. Este rasgo característico del artículo definido también recibe el nombre de condición de unicidad. 
Por lo tanto, podemos decir que el uso del artículo definido en español parte del conocimiento de la entidad por parte del hablante y sirve como garantía al oyente de que dicha entidad es accesible, es decir, es identificable. Como bien apunta Leonetti,

en algunos casos, dicha identificación (que consiste en acceder a una representación adecuada del referente) es inmediata; en otros, requiere la puesta en marcha de algún proceso inferencial por parte del oyente y la recuperación de ciertos supuestos contextuales implícitos (795).

De esta manera, la identificación del referente no es inmediata, y por lo tanto, la presencia del artículo definido permite que el oyente tenga conocimiento de la existencia del referente denotado por el sustantivo.

Así como es importante tomar en cuenta qué es el artículo definido en español, es importe saber cómo se expresa la definitud en la lengua materna de los hablantes que formaron parte de esta investigación, ya que aunque carezca de la categoría del artículo, eso no significa que no cuente con otros mecanismos para expresar la definitud del referente. En cuanto a la definitud en chino, primero es necesario aclarar a qué nos referimos cuando utilizamos el término chino.

Después de la familia indoeuropea, la familia sinotibetana es la segunda más grande en cuanto a número de hablantes. Esta familia se compone de dos grandes grupos de lenguas: el grupo sínico o chino y el grupo birmanotibetano. El grupo chino se compone de siete lenguas que son: mandarín北方 方言 [běifäng fāngyán]; wu 吴方言 [wú fāngyán]; xiang o hunan湘方言 [xiāng fängyán]; gan o Jiangxi 赣方言 [gàn fāngyán]; kejia o hakka 客家方言 [kèjia fängyán]; ye o cantonés 畧方言 [yuè fāngyán]; y min o fujian 闽方言 [m凶n fāngyán] (Cortés 2009:19-20).

Cabe señalar que dentro de la literatura del chino encontramos que a estas se le denominan dialectos. De esta manera, encontramos que varios autores definen al grupo chino como un grupo de ocho dialectos mutuamente ininteligibles a nivel oral (Li y Thompson 1981:2), (Norman 1988:2), (Yip 2006:1), (Cortés 2009:19). La razón por la que se usa el término dialecto y no lengua para referirse a cada una de las lenguas que conforman el grupo sínico tiene que ver con asuntos ajenos como: político-geográficos, culturales y de traducción.

Una vez que se ha presentado la situación de las lenguas que conforman el grupo chino, podemos decir que el término chino se usa de dos maneras. Por un lado, se utiliza, para denominar al conjunto de lenguas habladas por la etnia Han que constituye el $94 \%$ de la población de China. El otro $6 \%$ de la población china está compuesto por 55 minorías étnicas que cuentan con sus propias lenguas que no pertenecen a la familia sínica (Cortés 2009:17). Desde este punto 
de vista, el chino, denominado 汉语 [hànyǔ] que significa 'la lengua de los han', está conformado por las siete lenguas que mencionamos en un principio.

Por otro lado, el término chino se emplea para llamar a la lengua oficial de la República Popular de China. Es decir, de las ocho lenguas que conforman el chino, el mandarín hablado en Pekín la capital de China, no sólo es el que cuenta con el mayor número de hablantes, sino también es la lengua en la que se basó el gobierno de la República Popular China para establecer la lengua oficial del país en 1955 (Li y Thompson 1981:1). El término en chino que se utiliza para referirse al mandarín como lengua oficial en China continental es 普通话 [pǔtōnghuà] 'lengua común'; mientras que en Taiwán se le llama国语 [guóyǔ] 'lengua nacional'. Además, otro nombre para referirse al mandarín es el término antiguo y en desuso 官话 [guānhuà] que significa 'lengua de los oficiales' o'lengua de los mandarines' ya que en español, el término mandarín se refiere al hombre que tenía a su cargo el gobierno de una ciudad o la Administración de Justicia en China y en otros países asiáticos, de acuerdo con el Diccionario de la Real Academia Española.

A continuación presentaremos las características del chino más relevantes que nos ayudarán a entender cómo se expresa la definitud y la indefinitud en esta lengua. Asimismo, estas características nos darán un panorama de las diferencias o similitudes que existen entre el español y el chino.

\section{a) Es una lengua aislante}

El chino es el ejemplo prototípico de una lengua aislante. En el caso de los sustantivos, la unidad de la palabra equivale a un morfema que no puede analizarse en partes más pequeñas en donde podamos encontrar información sobre el género, el número, o el caso. Por lo tanto, a diferencia del español, los sustantivos en chino no tienen marca de género y número. Por ejemplo, el sustantivo 猫 $[m \bar{a} o]$ puede significar 'gato' o 'gatos', dependiendo del contexto.

El único caso en donde el chino marca pluralidad mediante un sufijo es a través del sufijo - 们 [-men] que sólo se utiliza en los pronombres personales y en los sustantivos que se refieren a personas:

$\begin{array}{cc}\text { Singular } & \text { Plural } \\ \text { 你 } & \text { 你们 } \\ \mathrm{n} \text { I } & \mathrm{n} \mathrm{I} \mathrm{men} \\ \text { 'tú' } & \text { 'ustedes' } \\ \text { Singular } & \text { Plural } \\ \text { 学生 } & \text { 学生们 } \\ \text { xuéshēng } & \text { xuéshēngmen } \\ \text { 'alumno' } & \text { 'alumnos' }\end{array}$

(2) 


\section{b) Es una lengua con clasificadores}

Los clasificadores, al igual que el artículo en español, dependen de un sustantivo. Mientras que el artículo solamente está vinculado con el sustantivo al que antecede en género y número, los clasificadores son una clase de palabra que mantiene una correlación semántica con el sustantivo al que se le asocia. Esto significa que los clasificadores dependen semánticamente del sustantivo porque denotan un rasgo característico percibido, o atribuido arbitrariamente, de la entidad con la que es relacionado (Luque 2004:28). De esta manera, los clasificadores sirven para ubicar a las entidades denotadas por los sustantivos como miembros de una categoría conceptual de acuerdo con sus características físicas. Cabe mencionar que las categorías conceptuales que conforman los clasificadores son el resultado de la conceptualización del mundo ya que dependen de cómo son percibidos los objetos por la sociedad china.

Por ejemplo, ¿en qué se parecen una perla y una estrella? Ya que la estrella es percibida como un objeto pequeño redondo y la perla tiene la misma forma, ante ambas entidades se utiliza el clasificador 颗 [kē]. Por lo tanto, los objetos que requieren este clasificador tienen la característica de ser pequeños y redondos como granos, por ejemplo: 牙齿 [yáchi] 'diente'; 葡萄 [pútao] 'uva'; 大豆 [dàdòu] 'frijol de soya'; 种子[zhǒngzi] 'semilla'; 子弹 [zìdàn] 'bala'; 行星 [xíngxīng] 'planeta' e incluso el sustantivo心 $[x \bar{i} n]$ 'corazón' utiliza este clasificador. Sin embargo, para referirse a entidades más pequeñas como米 [mì] 'arroz' o沙 [shā] 'arena', se prefiere el uso del clasificador 粒 [li] que también se refiere a granos más pequeños.

Los clasificadores en chino se utilizan ante el sustantivo cuando éste debe ser señalado por medio de los demostrativos这 [zhè] 'este' o 那 [nà] 'aquel' o cuando es contabilizado por medio de numerales. Por lo tanto, el clasificador puede ir encabezado por un demostrativo (4a), por un numeral (4b) o por un demostrativo y un numeral.

(4) a.

Demostrativo

这

zhè

Este

'Esta estrella'

b.

Numeral
-
yī
uno

'Una estrella'

$\begin{array}{cc}\text { Clasificador } & \text { Sustantivo } \\ \text { 颗 } & \text { 星星 } \\ \text { kē } & \text { xinngxing } \\ \mathrm{CL} & \text { estrella }\end{array}$

$\begin{array}{cc}\text { Clasificador } & \text { Sustantivo } \\ \text { 颗 } & \text { 星星 } \\ \text { kē } & \text { xīngxing } \\ \mathrm{CL} & \text { estrella }\end{array}$




\section{c) Es una lengua sin artículos}

En la gramática del chino no hay artículos (Chao 1991:37). Es decir, no hay una palabra que sirva como marca de que el objeto al que antecede es identificable para el oyente. Sin embargo, varios autores mencionan que el equivalente al artículo definido en chino son los demostrativos这 [zhè] 'este' y 那 [nà] 'ese' (Li y Thompson 1981:131-132).

Ciertamente, los demostrativos en chino sirven como una marca de definitud del referente ya que una de las funciones de los demostrativos, al igual que el artículo definido, es hacer referencia a una entidad única. Incluso, es posible sustituir un demostrativo por un artículo. Sin embargo, no siempre se puede sustituir el artículo por un demostrativo. Solamente llegan a ser intercambiables cuando el uso del artículo es deíctico y en el uso anafórico. De esta manera, los demostrativos en chino pueden interpretarse como artículo en los siguientes ejemplos.

(5)

$\begin{array}{cc}\text { Demostrativo } & \text { Clasificador } \\ \text { 这 } & \text { 个 } \\ \text { zhè } & \text { ge } \\ \text { este } & \mathrm{CL}\end{array}$

$\begin{array}{cc}\text { Sustantivo } & \text { Complemento } \\ \text { 家 } & \text { 很 漂亮 } \\ \text { jiā } & \text { hěn piàoliang } \\ \text { casa } & \text { muy bonita }\end{array}$

'Esta/La casa está muy bonita.'

Como ya se ha mencionado, una de las características que distingue al chino del español es la carencia de artículo definido. Sin embargo, el hecho de que el chino carezca de este determinante no quiere decir que el rasgo que aporta el artículo determinado, es decir, la definitud, no exista en este idioma. Al contrario, es un hecho que la referencialidad nominal, es decir, la capacidad de anclar los nombres a un objeto o a una persona con la realidad en el acto comunicativo constituye

un ámbito semántico fundamental de las lenguas naturales ya que nos informa del conocimiento que los hablantes tenemos sobre las entidades, lingüísticas y extralingüísticas, que nos rodean, $y$, en consecuencia, todas las gramáticas tienen expresiones y estrategias sintácticas diversas para la identificación del referente de una entidad y para orientar al oyente, o lector, en la adecuada comprensión de tales referentes (Company 2006:65). 
Así pues, ante la ausencia del artículo determinado, es importante ver las estrategias con las que cuenta el chino para indicar que un sustantivo es definido, es decir, es accesible e identificable al oyente.

En chino, la interpretación de las expresiones definidas e indefinidas se da por el orden de palabras. Cuando un SN está en posición preverbal es porque el objeto designado por el sintagma es conocido por el hablante e identificable para el oyente. En cambio, si el SN está en posición posverbal seguramente se menciona por primera vez en el discurso y por lo tanto es desconocido y no identificable para el oyente. De esta manera, los SSNN en posición preverbal se interpretan como sintagmas definidos mientras que los sintagmas posverbales son indefinidos (Cheng y Sybesma 1999:509) (Li y Thompson 1981:85) (Chao 1991:37) (Yip 2006:14).

Verbo + Sintagma Nominal $=$ Sintagma nominal indefinido

(6) a.

$\begin{array}{ccc}\text { Sujeto } & \text { Verbo } & \text { SN indefinido } \\ \text { 我 } & \text { 买 } & \text { 书 } \\ \text { w ǒ } & \text { măi } & \text { shū } \\ \text { yo } & \text { comprar } & \text { libro }\end{array}$

'Yo compro un libro/libros

Sintagma Nominal + Verbo $=$ Sintagma nominal definido

$\begin{array}{ccc}\text { b. Sintagma nominal definido } & \text { Verbo } & \text { Pronombre interrogativo } \\ \text { 书 } & \text { 在 } & \text { 哪里? } \\ \text { shū } & \text { zài } & \text { nă li } \\ \text { libro } & \text { estar } & \text { dónde }\end{array}$

De esta manera, el chino cuenta con la particularidad de que el SN escueto, es decir, que no está encabezado por ningún tipo de determinante, puede recibir interpretación definida o indefinida gracias al lugar que ocupa en la oración. Además, debido a que los sustantivos contables no cuentan con marca de número, pueden interpretarse en singular o plural, por lo que pueden referir a una entidad individualizada o a la acumulación de ellas. 
LA CONSTRUCCIÓN把 $[B \ddot{A}]$

A diferencia del español, el chino cuenta con una estructura en donde los sintagmas nominales definidos se anteponen al verbo por medio de la partícula 把 $[b a ̆]$. Las condiciones que se deben cumplir para usar esta construcción son las siguientes:

1. El sintagma nominal debe ser definido porque la entidad que lo constituye cumple con la condición de unicidad. Es decir, el objeto referido puede ser identificado manera unívoca por el oyente.

2. El verbo de la oración nunca debe ir solo, es decir, debe tener otros elementos que complementen su significado

(7) a.

Sujeto BA SN definido Verbo Complemento de

我儿子

yo hijo

b ă

水
shu Ǐ
agua

喝
Hē
tomar
resultado

'Mi hijo se tomó el agua.'

b.

\begin{tabular}{ccccc} 
Agente & BA & SN definido & Verbo & \multicolumn{2}{c}{ C. de resultado } \\
她 & 把 & 这 封 信 & 翻译 & 成 日语 \\
tā & b à & zhè fēng xìn & fānyì & chéng rìy ǔ \\
ella & BA & este $C L$ carta & traducir & cambiar japonés
\end{tabular}

'Ella traduce esta carta al japonés.'

Como podemos apreciar en los ejemplos, el sintagma nominal definido antecede al verbo de la oración, y a su vez, la partícula把 [bă] encabeza al sintagma nominal. De esta manera, gracias a la partícula把 [bă] el orden SVO cambia a SOV.

\section{DESCRIPCIÓN DEL CORPUS}

El corpus que analizaremos en este trabajo está conformado por las producciones de 27 informantes cuya lengua materna es el chino. De acuerdo al momento en el que fue tomada la muestra, contamos con informantes que tienen desde un mes hasta dieciséis años residiendo en la ciudad de México. 
De los 27 informantes, 20 de ellos son estudiantes de español del Centro de Enseñanza para Extranjeros (CEPE), sede Ciudad Universitaria. En total, contamos con 9 estudiantes de básico 1; 6 estudiantes de básico 2; 1 estudiante de básico 3 ; y 4 estudiantes de intermedio 1. Los otros 7 informantes ya concluyeron sus estudios de español y tienen un tiempo significativo de exposición a la lengua debido al trabajo y a la vida que llevan en México. Los informantes que conforman este grupo son: dos profesoras de chino; una abogada; un profesor de microbiología; una estudiante universitaria; y dos comerciantes.

Como podemos ver, el grupo representativo que conforma este corpus es heterogéneo con el fin de describir el uso del artículo en un grupo diverso. Además, este corpus cuenta con la ventaja de que podemos observar el uso del artículo en diferentes etapas de la adquisición en un ambiente de instrucción formal y también podremos analizar qué pasa con esta categoría cuando la instrucción formal ha terminado o ha sido interrumpida y el sujeto sigue haciendo uso de la lengua debido al ambiente laboral y a sus necesidades para vivir en México.

\section{INSTRUMENTO}

La habilidad lingüística que tomamos en cuenta para la conformación de nuestro corpus es la expresión escrita. Por lo tanto, la técnica que utilizamos para recopilar los datos tiene como fundamento la producción escrita. De esta manera, el instrumento que empleamos consta de dos partes: la primera, es una redacción que tiene como tema La vida en México; y la segunda, consiste en la traducción inversa' de 25 oraciones.

De las 25 oraciones, 24 son de mi autoría y una la tomé de la Gramática práctica del chino de Liljiana Arsovska. Estas oraciones fueron preparadas con el fin complementar los datos del corpus y poner especial atención en usos del artículo que pueden ser conflictivos para los informantes, y por lo tanto pueden ser evitados en la redacción semi-controlada.

\section{Procedimiento de APLICACIÓN DEL INSTRUMENTO}

Las pruebas que conforman el corpus se recolectaron de enero a marzo del año 2011. Cabe decir, que el corpus está conformado con los datos de las personas que tuvieron la disponibilidad de cooperar con esta investigación. En el caso de

1 La traducción inversa consiste en la traducción de la lengua materna del aprendiz, que es el chino, a la lengua meta, el español. 
los estudiantes del CEPE, localicé a los alumnos chinos fuera de los salones de clase, durante su descanso, y ellos a su vez, me presentaban a otros alumnos. De esta manera, apliqué el instrumento fuera de los horarios de clase, bajo mi supervisión. En el caso de los otros informantes, recurrí a contactos personales como mis maestras de chino del CELE y del Instituto Confucio de la UNAM; así como el maestro En Tao, profesor de la Escuela Nacional de Ciencias Biológicas del Instituto Politécnico Nacional, y su familia.

Además, acudí al centro de la Ciudad de México, específicamente a la calle Apartado, ubicada entre la calle del Carmen y la calle República de Perú, colonia Centro, delegación Cuauhtémoc, en donde se encuentra el edificio llamado Centro de Mayoreo que concentra una gran cantidad de comercios manejados por chinos. La recolección de datos en este lugar fue muy interesante porque descubrí un punto que concentra y representa las posibilidades de adquisición de una lengua. Por un lado encontramos chinos que llevan muchos años viviendo en México y no hablan nada de español; encontramos chinos que aprendieron español ahí, en inmersión; encontramos alumnos y exalumnos del CEPE; encontramos chinos que aprendieron español en otros países de habla hispana; encontramos una generación de jóvenes que llegaron de China y han aprendido español en la secundaria, preparatoria o universidad y por lo tanto son el vínculo entre sus padres y todo lo que tenga que ver con México y los mexicanos; y finalmente encontramos nuevas generaciones de chinos nacidos en este país que hablan español desde pequeños y hablan con sus familiares en cantonés o en la lengua específica de su región pero no hablan mandarín. Relato esta experiencia para señalar el campo inexplorado con el que contamos para analizar la situación lingüística de la adquisición del español como segunda lengua en el grupo particular de chinos que residen en México, situación que puede ser aprovechada y retomada para futuras investigaciones.

Retomando la explicación de la aplicación de mi instrumento, debo comentar que la aplicación de los cuestionarios en este lugar no fue fácil. En primer lugar, uno, como investigador, llega a su lugar de trabajo y si están muy ocupados, no tienen tiempo para colaborar con la investigación. Por otro lado, un factor importante que obstaculiza la obtención de los datos consiste en que hay chinos que sí hablan español pero no quieren contestar el cuestionario debido a que no lo han estudiado formalmente en una escuela y les da pena o les intimida un poco la formalidad y la extensión del documento, sin embargo, me pusieron en contacto con las personas que ellos consideran que era apta para contestar el cuestionario, situación que hace que la obtención de las encuestas se prolongue por más tiempo. 
Metodología

Para el análisis del corpus utilizamos la metodología de Análisis de Errores. Este método nos ayudó a describir la actuación lingüística de un grupo representativo de hablantes con el fin de generalizar las conclusiones a las que llegamos, o también nos ayudarán a formular nuevas hipótesis que podrán ser abordadas en futuras investigaciones.

Tipología de errores en el uso del artículo definido

a) Errores de omisión:

- $\varnothing$ señor Pérez es médico (Informante de básico 3);

- yo trabajo en $\varnothing$ UNAM (Informante de Básico 1);

- Ø Español me ayuda buscar trabajo (Informante de Básico 2).

b) Errores de adición de otros elementos ante el artículo:

-no hay tarea en el viernes (Informante de Intermedio 1);

-pedí mi la cerular. (Informante no estudiante).

c) Errores de falsa elección:

- ella es una estudiante más bella en su grupo;

- Trajo frutas mi papá en lunes. (Informante de Intermedio 1).

d) Error de número en el artículo en plural ante sustantivos no cantables cuando debe ser singular:

- poner los nieves en la caja, por favor. (Informante de Básico 3);

- Las comidas mexicanas son ricos. (Informante de Intermedio 1).

RESUltAdOS DEL ANÁLISIS

De acuerdo con la clasificación de los errores en el uso del artículo y los aciertos obtuve los siguientes porcentajes de errores y aciertos:

Tabla 1

Total de aciertos y errores en el uso del artículo

$\begin{array}{ccc}\text { Errores } & 210 & 27 \% \\ \text { Aciertos } & 557 & 73 \% \\ \text { Total } & 767 & 100 \%\end{array}$




\section{Tabla 2}

Total de aciertos y errores en la ausencia del artículo definido

$\begin{array}{ccc}\text { Aciertos } & 251 & 71 \% \\ \text { Errores } & 102 & 29 \% \\ \text { Total } & 353 & 100 \%\end{array}$

Como conclusión, de acuerdo con los resultados que se obtuvieron del análisis de errores, los informantes dominan el uso del artículo definido en contextos donde la presencia de este determinante es obligatoria, es decir, no hay vacilación entre su ausencia o no. Este tipo de estructuras se encuentran en la expresión de la hora y el momento del día.

Un hallazgo de esta investigación que debemos considerar consiste en que los informantes tienden a pluralizar los sustantivos no contables referentes a la acumulación de partículas tanto cuando llevan artículo definido, como cuando no lo llevan, por ejemplo: La botella está llena de las piedras y arenas (Informante de Intermedio 1); En Cancún las arenas son blancas (Informante no estudiante).

Con respecto al contraste entre los dos grupos de informantes que conforman el corpus, es decir, los informantes que están expuestos al español en un ambiente escolarizado y los que ya concluyeron o abandonaron sus estudios, radican en México y tienen que hacer uso del español, encontramos que éstos últimos cometen los mismos errores que los informantes que estudian en los niveles básicos 1 y 2 . De esta manera, vemos, que si no se está en un ambiente escolarizado, la omisión del artículo tiende a fosilizarse como en el siguiente ejemplo: $\varnothing$ Señor Pérez es médico.

Considero que a partir de este trabajo, una brecha de investigación que surgió fue la recategorización de los sustantivos contables y no contables. Asimismo, es importante contemplar la elaboración de materiales para la enseñanza y práctica de este tema.

Esperamos que este trabajo contribuya a un mejor conocimiento del español como lengua extranjera en hablantes cuya lengua materna es el chino. 
BIBLIOGRAFÍA

AıcINA Franch, Juan y José Manuel Blecua. (1988) Gramática española, Barcelona: Ariel.

CHAO, Yue Ren. (1991) Sinopsis sintética de la gramática del chino moderno Cuatro estudios sobre la gramática del chino, R. Maeth (comp.), México: El Colegio de México.

CHENG, Lisa y Rint Sybesma. (1999) "Bare and not-so-bare nouns and the structure of NP", Linguistic Inquiry, 30 (4), Estados Unidos: MIT Press Journals, pp. 509-542.

Company Company, Concepción. (2006) Persistencia referencial, accesibilidad y tópico La semántica de la construcción artículo+posesivo+sustantivo en el español medieval, Revista de Filología Española, LXXXVI, (1), pp. 65-103.

CORTÉs Moreno, Maximiano. (2009) Fonología china, Barcelona: Herder.

GILI Gaya, Samuel. (1980) Curso superior de sintaxis española, Barcelona: Biograf. Leonetti Jungl, Manuel. (1990) El artículo y la referencia, Madrid: Taurus. . (1999a) El artículo. Gramática descriptiva de la lengua española, I. Bosque y V. Demonte (dirs.), Madrid: Espasa Calpe, pp. 787-890.

LI, Charles N. y Sandra Thompson. (1981) Mandarin Chinese: a functional reference grammar, Berkeley: University of California Press.

Lin, Tzu Jun. (2003). La adquisición y el uso del artículo por alumnos chinos. Tesis de doctorado. [en línea]. Universidad de Alcalá. Recuperado 10 de agosto de 2012.<http://www.mecd.gob.es/dctm/redele/ MaterialRedEle/Biblioteca/2005_BV_04/2005_BV_04_16Tzu-Ju. pdf?documentld=0901e72b80e3cea2 >

Luque Durán, Juan de Dios. (2002) Aspectos universales y particulares del léxico de las lenguas del mundo. Granada: Método.

Norman, Jerry. (1988) Chinese, Cambridge: Cambridge University Press.

YIP, Po-Ching y Don Rimmington. (2006) Chinese: an essential grammar, New York: Routledge. 
